\title{
DYNAMICS OF NON-AUTONOMOUS REACTION-DIFFUSION EQUATIONS IN LOCALLY UNIFORM SPACES
}

\author{
Gaocheng Yue - Chengkui Zhong
}

\begin{abstract}
In this paper, we first prove the well-posedness for the nonautonomous reaction-diffusion equations on the entire space $\mathbb{R}^{N}$ in the setting of locally uniform spaces with singular initial data. Then we study the asymptotic behavior of solutions of such equation and show the existence of $\left(H_{U}^{1, q}\left(\mathbb{R}^{N}\right), H_{\phi}^{1, q}\left(\mathbb{R}^{N}\right)\right)$-uniform(w.r.t. $\left.g \in \mathcal{H}_{L_{U}^{q}\left(\mathbb{R}^{N}\right)}\left(g_{0}\right)\right)$ attractor $\mathcal{A}_{\mathcal{H}_{L_{U}^{q}\left(\mathbb{R}^{N}\right)}\left(g_{0}\right)}$ with locally uniform external forces being translation uniform bounded but not translation compact in $L_{b}^{p}\left(\mathbb{R} ; L_{U}^{q}\left(\mathbb{R}^{N}\right)\right)$. We also obtain the uniform attracting property in the stronger topology.
\end{abstract}

\section{Introduction}

In this paper, we consider the long-time dynamical behavior of solutions for the following non-autonomous reaction-diffusion equations on $\mathbb{R}^{N}$ :

$$
u_{t}-\Delta u+f(x, u)=g(x, t), \quad \text { in } \mathbb{R}^{N} \times[\tau, \infty),
$$

with the initial condition

$$
u(x, \tau)=u_{\tau}(x), \quad x \in \mathbb{R}^{N},
$$

where $f: \mathbb{R}^{N} \times \mathbb{R} \rightarrow \mathbb{R}$ is a suitable continuous function and $g$ is given the external force.

2010 Mathematics Subject Classification. Primary: 35K57, 35B40; Secondary: 35B41.

Key words and phrases. Reaction-diffusion equations, uniform attractors, locally uniform spaces.

The first author is supported by NSF of China under Grant 11501289, and by the Fundamental Research Funds for the Central Universities, No. NS2014075. 
Our goal is to prove that under suitable conditions there exist the uniform attractor of reaction-diffusion equation with singular initial data and forcing term $g(x, t)$ being translation uniform bounded but not translation compact in an appropriate sense.

The nonlinear term $f$ is assumed to satisfy the following assumptions:

Assumption I. Assume that $f$ satisfies $f(x, 0)=0$ and there exist constants $C$ and $\rho$ with $C>0, \rho>1$ such that

$$
|f(x, u)-f(x, v)| \leq C|u-v|\left(1+|u|^{\rho-1}+|v|^{\rho-1}\right), \quad \text { for all } u, v \in \mathbb{R} .
$$

Assumption II. There exist two positive constants $\mu_{0}, \mu_{2}$ such that

$$
-\mu_{0} s^{2}-\mu_{2}|s| \leq s f(x, s), \quad \text { for all } s \in \mathbb{R}, x \in \mathbb{R}^{N} .
$$

We furthermore assume that the external force $g(\cdot, t) \in \dot{L}_{U}^{q}\left(\mathbb{R}^{N}\right)$ with $1<$ $q<N$ for almost every $t \in \mathbb{R}$ and $g$ has finite norm in the space $L_{b}^{p}\left(\mathbb{R}, \dot{L}_{U}^{q}\left(\mathbb{R}^{N}\right)\right)$ with $p>2$, i.e.

$$
\|g\|_{L_{b}^{p}\left(\mathbb{R}, \dot{L}_{U}^{q}\left(\mathbb{R}^{N}\right)\right)}^{p}=\sup _{t \in \mathbb{R}} \int_{t}^{t+1}\|g(\cdot, s)\|_{\dot{L}_{U}^{q}\left(\mathbb{R}^{N}\right)}^{p} d s<+\infty .
$$

The main contribution of the present paper is to extend the external force to the case where $g(x, t)$ is considered in much larger space $L_{b}^{p}\left(\mathbb{R}, \dot{L}_{U}^{q}\left(\mathbb{R}^{N}\right)\right)$ and with weaker assumptions.

The long-time behavior of the solutions of (1.1) is of great current interest. It is well-known that this behavior of solutions of such equations arise from mathematical physics can be described as the existence of the so-called attractors of the corresponding semigroups (or process). In particular, when the domain is bounded, the global attractor of such problems have been extensively studied by using many different methods in the literature (see, e.g. [6], [13], [19], [23], [29], [32], [35], [37] and the references therein).

In contrast to this, the case of unbounded domain becomes much more difficulty and some of methods for bounded domains are on longer valid. The main difficulty lies in the absence of the standard Sobolev compact embedding.

On the other hand, the disadvantage of the standard Lebesgue spaces $L^{p_{1}}\left(\mathbb{R}^{N}\right)$ $\left(1 \leq p_{1}<\infty\right)$ is that it does not consider the behavior of the solutions for large spatial values and the family of such spaces are not nested. In order to overcome these difficulties, one should introduce an appropriate functional spaces to study these partial differential equations. With respect to the use of weighted Sobolev spaces, one of the pioneer works is in [7], where Babin and Vishik for the first time introduced weighted Sobolev space as the phase space and showed, under appropriate assumptions, the existence of global attractors for parabolic type evolutionary equations on unbounded domain, however, which requires the initial data and forcing term belonging to the corresponding weighted spaces (see 